\title{
ESTATUTO DA CRIANÇA E DO ADOLESCENTE E DIREITOS HUMANOS SEXUAIS E REPRODUTIVOS NA FORMAÇÃO DE ENFERMEIROS E MÉDICOS
}

Jéssica do Nascimento Xavier ${ }^{1}$, Mariana Nogueira Marconsin ${ }^{1}$, Mariane Marçal do Nascimento ${ }^{1}$, Leila Adesse ${ }^{2}$, Lia Silveira ${ }^{3}$, Adriana Lemos ${ }^{4}$

\begin{abstract}
RESUMO: Esta pesquisa teve por objetivo identificar conteúdos referentes aos Direitos Humanos Sexuais e Reprodutivos e Estatuto da Criança e do Adolescente nos programas de disciplinas das áreas de Saúde da Mulher, Saúde do Adolescente e Saúde Coletiva dos cursos de graduação em Enfermagem e Medicina das Universidades Públicas do Estado do Rio de Janeiro. Foram analisados 30 programas de disciplinas da graduação em Enfermagem e 18 de Medicina através de matriz de análise sob a técnica de análise temática. O tema Direitos Sexuais e Reprodutivos está presente apenas no item Referência Bibliográfica de um programa, e o Estatuto da Criança e do Adolescente consta nas Referências de três programas, apenas do curso de Enfermagem. Identificou-se não padronização e bibliografia desatualizada nos programas dos cursos deste estudo com possíveis prejuízos à formação profissional.
\end{abstract}

DESCRITORES: Educação em enfermagem; Direitos sexuais e reprodutivos; Saúde da mulher; Saúde do adolescente; Saúde coletiva.

\section{STATUTE OF THE CHILD AND ADOLESCENT AND HUMAN, REPRODUCTIVE AND SEXUAL RIGHTS IN THE TRAINING OF NURSES AND DOCTORS}

\begin{abstract}
This research aimed to identify content referent to Human, Reproductive and Sexual Rights and the Statute of the Child and Adolescent in the programs of courses in the areas of Women's Health, Adolescent Health and Collective Health of the undergraduate Nursing and Medicine courses at the Public Universities of the State of Rio de Janeiro. 30 programs from undergraduate nursing courses, and 18 from Medicine, were analyzed, by using the evaluation matrix with the technique of thematic analysis. The topic of Sexual and Reproductive Rights is present only in the item 'Bibliographic Referencing' of one program, and the Statute of the Child and Adolescent is to be found in the References of three programs, and only in the Nursing course. Lack of standardization and a bibliography which was not up-to-date were identified in the courses' programs in this study, with possible harm to professional training.

DESCRIPTORS: Nursing education; Sexual and reproductive rights; Women's health; Adolescent health; Collective health.

\section{ESTATUTO DEL NIÑO Y DEL ADOLESCENTE Y DERECHOS HUMANOS SEXUALES Y REPRODUCTIVOS EN LA FORMACIÓN DE ENFERMEROS Y MÉDICOS}

RESUMEN: Esta investigación tuvo la finalidad de identificar contenidos sobre Derechos Humanos Sexuales y Reproductivos y Estatuto del Niño y del Adolescente en los programas de disciplinas de áreas de Salud de la Mujer, Salud del Adolescente y Salud Colectiva de los cursos de graduación en Enfermería y Medicina de las Universidades Públicas del Estado de Rio de Janeiro. Fueron analizados 30 programas de disciplinas de graduación en Enfermería y 18 de Medicina a través de matriz de análisis bajo la técnica de análisis temático. El tema Derechos Sexuales y Reproductivos está presente solamente en el ítem Referencia Bibliográfica de un programa, y el Estatuto del Niño y del Adolescente está en las Referencias de tres programas, solamente del curso de Enfermería. Fue identificada una no patronización, así como bibliografía desactualizada en los programas de los cursos de este estudio con posibles perjuicios a la formación profesional. DESCRIPTORES: Educación en enfermería; Derechos sexuales y reproductivos; Salud de la mujer; Salud del adolescente; Salud colectiva.

\footnotetext{
${ }^{1}$ Acadêmica da Escola de Enfermagem Alfredo Pinto da Universidade Federal do Estado do Rio de Janeiro - UNIRIO. Bolsista de Iniciação Científica. Membro do Grupo de Pesquisa Enfermagem e População: Atitudes, Conhecimentos e Práticas em Saúde.

${ }^{2}$ Médica Sanitarista. Doutoranda em Saúde da Mulher e da Criança pelo Instituto Fernandes Figueira FIOCRUZ. Diretora da ONG AADS. ${ }^{3}$ Psicóloga. Mestre em Tecnologia Educacional nas Ciências da Saúde. Tecnóloga educacional da Universidade Federal do Rio de Janeiro. ${ }^{4}$ Enfermeira Sanitarista. Doutora em Saúde Coletiva. Professora da Escola e do Programa de Pós-Graduação em Enfermagem da Escola de Enfermagem Alfredo Pinto da UNIRIO. Membro do Grupo de Pesquisa Enfermagem e População: Atitudes, Conhecimentos e Práticas em Saúde e do Núcleo de Pesquisa Estudos e Experimentação em Enfermagem na Área da Mulher e da Criança.
} 


\section{INTRODUÇÃO}

A prática profissional em saúde é permeada por questões que envolvem os direitos sexuais e reprodutivos, desta forma, tratar de tal questão na formação é necessário para a construção de boas práticas profissionais no que tange a promoção de uma atenção integral à saúde. Os direitos sexuais e reprodutivos (DSR) têm status de direitos humanos, portanto abrangem toda $\mathrm{e}$ qualquer pessoa e devem ser reconhecidos no mesmo patamar dos direitos sociais e econômicos ${ }^{(1)}$.

Grande parte dos profissionais de saúde "não reconhecem os direitos reprodutivos como parte integrante dos direitos humanos fundamentais" "(2:18), e o mesmo se apresenta para o reconhecimento dos direitos sexuais. A promoção e garantia desses direitos está diretamente ligada ao trabalho dos profissionais de saúde, de modo que, dependendo de seu comportamento frente ao atendimento à clientela, tal garantia pode ser comprometida ${ }^{(3)}$.

O discurso predominante sobre a adolescência e sexualidade é marcado por uma visão biomédica onde a ideia de risco ou vitimização é mais presente. Logo, a sexualidade como algo positivo em si e, sobretudo, como um direito, está pouco presente nos documentos e políticas que envolvem este grupo. Além disso, há discrepância entre o que é recomendado nas políticas norteadoras e a prática cotidiana dos serviços.

A Política Nacional de Atenção Integral à Saúde de Adolescentes e Jovens, por exemplo, que está fundamentada nos direitos humanos e tem como pilares normativos a Constituição Federal e o Estatuto da Criança e do Adolescente (ECA), reconhece crianças e adolescentes como sujeitos sociais, portadores de direitos e garantias próprias ${ }^{(4)}$. No entanto, tal grupo encontra dificuldades no acesso a determinados serviços de saúde, sobretudo nas práticas de cuidado à saúde sexual e saúde reprodutiva. $\mathrm{O}$ adolescente não se vê no espaço do serviço de saúde pois, em geral, este é um espaço para mulheres e crianças, cujo foco do cuidado não está na prevenção ou na saúde e sim em doenças.

O Ministério da Saúde, no aprofundamento da discussão sobre formação profissional em saúde atrelada à renovação do modelo assistencial e à implementação de políticas articuladas entre universidade, serviço e comunidade, considera o Sistema Único de Saúde (SUS) como superfície de emergência de novas demandas que alavancam as mudanças necessárias para a transformação da educação médica ${ }^{(5)}$. Esta discussão também se aplica às mudanças na formação do profissional de enfermagem.
As Diretrizes Curriculares Brasileiras apresentam um conjunto de conteúdos essenciais para a formação no nível de graduação, dentre eles, inclui-se: a compreensão dos determinantes sociais, culturais, comportamentais, psicológicos, ecológicos, éticos e legais do processo saúde-doença. O Artigo $3^{\circ}$ define o perfil do egresso/profissional como generalista, humanista, crítico e reflexivo, com conhecimentos que permitam exercer seus serviços dentro dos mais altos padrões de qualidade e dos princípios da ética/bioética, com clareza sobre sua responsabilidade pela atenção integral à saúde, considerando que sua prática não se encerra com $\mathrm{o}$ ato técnico ${ }^{(6)}$. Os referidos determinantes têm relação direta com os direitos humanos sexuais e reprodutivos (DHSR). É necessário que o profissional de saúde deixe de desempenhar um papel apenas técnico e assistencial e passe a considerar a saúde como um direito social $\mathrm{e}$, portanto, humano, assegurando a integralidade da atenção e aderindo à sua prática as políticas de saúde vigentes, dentre elas as de saúde sexual e reprodutiva.

Desta forma, a presente pesquisa visa contribuir para o processo de mudança curricular para formar enfermeiros e médicos que incorporem, em suas práticas de atenção à saúde sexual e reprodutiva de mulheres e de adolescentes a perspectiva dos DHSR e dos princípios do Estatuto da Criança e do Adolescente. Tem, ainda, por objetivo identificar os conteúdos referentes aos DHSR e ao Estatuto da Criança e do Adolescente nos programas de disciplinas das áreas de Saúde da Mulher e da Saúde do Adolescente dos cursos de graduação em Enfermagem e Medicina das Universidades Públicas do Estado do Rio de Janeiro.

\section{MÉTODO}

Constitui-se de uma pesquisa de natureza descritiva com caráter documental. O cenário foram quatro cursos de Enfermagem e três de Medicina de instituições públicas do Estado do Rio de Janeiro.

Os programas de disciplinas das áreas de Saúde da Mulher, da Saúde do Adolescente e da Saúde Coletiva foram solicitados através de contato telefônico com os coordenadores dos cursos de Enfermagem e de Medicina. Após assinatura do termo de autorização estes foram obtidos, pessoalmente e/ou por e-mail, via coordenação e/ou secretarias dos cursos. O período de busca e recebimento dos referidos documentos, ou seja, os programas das disciplinas do currículo vigente, ocorreu entre o segundo semestre de 2010 e o primeiro semestre de 2011. Os cursos de Enfermagem disponi- 
bilizaram um total de 44 programas e os de Medicina, 47 programas.

Adotou-se como critérios de inclusão/exclusão dos programas na pesquisa: carga horária teórica ou teórico/prática e conteúdos relevantes ao objeto de estudo como, por exemplo, sexualidade, gênero, direito, foram inclusos. Os programas com carga horária exclusivamente prática e conteúdos relacionados apenas à Saúde de Criança, não abordando o Adolescente, foram excluídos do corpo de análise. Ao final desse processo, 30 programas de Enfermagem foram selecionados e 18 de Medicina. Os Cursos de Enfermagem foram denominados de EK, EX, EY e EZ e os de Medicina MK, MX e MY.

Priorizaram-se as disciplinas compreendidas na rede/matriz curricular obrigatória dos cursos. Porém, recebemos um programa de Enfermagem e um de Medicina que se encaixavam no quadro de disciplinas não obrigatórias e apresentavam conteúdo relacionado à pesquisa, sendo, desta forma, incluídas na análise.

Para análise documental utilizamos os programas de disciplinas e foi elaborada uma Matriz de Análise composta pelos itens: Ementa, Objetivos, Conteúdo Programático e Referências Bibliográficas. Utilizou-se a técnica de análise de conteúdo em sua modalidade temática ${ }^{(7)}$, abrangendo as seguintes fases: pré-análise, onde os programas foram separados por área; exploração do material, por meio da leitura exaustiva dos documentos; tratamento dos resultados obtidos, com a construção da matriz de análise e, por último, a interpretação com base no material bibliográfico adotado.

Cabe esclarecer que esta pesquisa foi aprovada, obtendo o parecer número 025/2010 do Comitê de Ética e Pesquisa da Universidade Federal do Rio de Janeiro.

\section{RESULTADOS}

Primeiramente descrevemos os resultados dos Cursos de Graduação em Enfermagem. Foram analisados 9 programas da área da Saúde da Mulher; 5 da Saúde do Adolescente e 16 programas da Saúde Coletiva. Os termos 'Direitos Humanos Sexuais e Reprodutivos' e os relativos ao Estatuto da Criança e do Adolescente se fazem presentes apenas no item referências bibliográficas de dois cursos. Por conta disso se procurou temas relacionados aos objetos do estudo. A presença dos temas descritos ao longo das ementas, objetivos, conteúdos programáticos e referências foram contabilizados originando a tabela 1.
Tabela 1 - Relação de temas encontrados nos programas de disciplinas por Curso de Enfermagem. Rio de Janeiro, 2011

\begin{tabular}{|c|c|c|c|c|c|}
\hline Temas Encontrados & EK & EX & EY & $\mathbf{E Z}$ & Total \\
\hline Políticas Públicas & 17 & 6 & 4 & 4 & 31 \\
\hline SUS & 12 & 0 & 0 & 5 & 17 \\
\hline $\begin{array}{l}\text { Integralidade da } \\
\text { Assistência }\end{array}$ & 1 & 4 & 0 & 2 & 7 \\
\hline $\begin{array}{l}\text { Direitos Humanos } \\
\text { Sexuais e Reprodutivos }\end{array}$ & 1 & 0 & 0 & 0 & 1 \\
\hline $\begin{array}{l}\text { Estatuto da Criança e do } \\
\text { Adolescente }\end{array}$ & 2 & 0 & 1 & 0 & 3 \\
\hline Gênero/Sexualidade & 0 & 0 & 2 & 1 & 3 \\
\hline $\begin{array}{l}\text { Ações/ Práticas } \\
\text { Educativas }\end{array}$ & 7 & 3 & 0 & 4 & 14 \\
\hline DST/AIDS & 2 & 0 & 1 & 1 & 4 \\
\hline Planejamento Familiar & 3 & 0 & 1 & 0 & 4 \\
\hline $\begin{array}{l}\text { Humanização da } \\
\text { Assistência }\end{array}$ & 2 & 1 & 0 & 0 & 3 \\
\hline Direito à Saúde & 7 & 0 & 1 & 2 & 10 \\
\hline Direito das Mulheres & 1 & 1 & 1 & 0 & 3 \\
\hline Direito dos Adolescentes & 0 & 0 & 0 & 0 & 0 \\
\hline Aborto & 2 & 0 & 0 & 0 & 2 \\
\hline PNAISM & 1 & 0 & 2 & 0 & 3 \\
\hline PAISM & 2 & 0 & 2 & 1 & 5 \\
\hline PROSAD & 1 & 0 & 0 & 1 & 2 \\
\hline Pacto pela Saúde & 0 & 0 & 0 & 0 & 0 \\
\hline Lei Orgânica da Saúde & 1 & 0 & 0 & 2 & 3 \\
\hline Constituição Federal & 4 & 0 & 0 & 0 & 4 \\
\hline Total & 66 & 15 & 15 & 23 & 119 \\
\hline
\end{tabular}

Os 20 temas listados foram encontrados 119 vezes nos 30 programas analisados. O tema DHSR não está presente em nenhum dos programas especificamente, a não ser pela referência de uma publicação do Ministério da Saúde sobre DSR em apenas um programa do curso EK. O ECA está presente apenas nas referências de três programas, dois do curso EK e um do curso EY.

No curso EK foram analisados dois programas de Saúde da Mulher e 8 de Saúde Coletiva, apresentando 66 vezes a maioria dos temas listados. Apesar de não apresentar nenhum programa referente à área Saúde do Adolescente, dois programas continham o ECA em suas referências: um da área de Saúde da Mulher que abordava também conteúdos referentes à Saúde da Criança e um programa da área de Saúde Coletiva, este trata da temática Adolescente em seu conteúdo, com tópicos sobre promoção da saúde, práticas educativas e a aplicabilidade dos programas governamentais voltados para este grupo.

Cogitare Enferm. 2013 Jan/Mar; 18(1):64-70 
Dos programas do curso EX, dois são da área de Saúde da Mulher, um de Saúde do Adolescente e um de Saúde Coletiva, onde os temas listados aparecem 15 vezes. No curso EY, com três programas de Saúde da Mulher, um de Saúde do Adolescente e três de Saúde Coletiva, o tema Gênero/Sexualidade é citado duas vezes nas referências de um programa de Saúde da Mulher. O mesmo programa cita em sua ementa e nos objetivos a Política Nacional de Atenção Integral à Saúde da Mulher (PNAISM), elaborada em 2004. No entanto, em suas Referências apresenta apenas o Programa de Assistência Integral à Saúde da Mulher (PAISM), elaborado pelo Ministério da Saúde em 1983 e publicado em 1984. O ECA está presente nas referências do único programa de Saúde do Adolescente. Já o curso EZ tem dois programas na área de Saúde da Mulher, três de Saúde do Adolescente e quatro na de Saúde Coletiva, totalizando 9 programas analisados.

Entretanto, ao compararmos a quantidade de vezes em que os mesmos temas são encontrados somente nas referências dos programas analisados, o número é reduzido em mais da metade. Vemos a necessidade de atualização dos programas, já que a maioria foi publicada na década de 1990 e há uma carência na indicação de publicações acerca dos conteúdos apresentados. A presença dos temas cai de 119 para 48, pois nem todos os cursos indicam documentos sobre os temas mencionados em suas referências.

As publicações que estão relacionadas ao DHSR são escassas e encontradas somente em dois cursos. Livros sobre Gênero/Sexualidade são encontrados apenas na curso EY. O PAISM é citado nos cursos EK e EY. Já o PNAISM e a Constituição Federal aparecem somente no curso EK, assim como publicações sobre aborto, humanização e DST/AIDS.

Observa-se que a distribuição dos temas não ocorre de forma uniforme. Nota-se também que os temas mais encontrados durante a análise dizem respeito à área de Saúde Coletiva, onde o foco da maioria dos programas dessa área se baseava na atuação do enfermeiro na atenção primária em saúde. Entretanto, a Lei Orgânica de Saúde, referência fundamental para a área, foi observada somente em dois programas de dois cursos de Enfermagem.

Nos programas das disciplinas do curso de Medicina, foram seis programas de Saúde da Mulher, quatro de Saúde do Adolescente e 8 programas de Saúde Coletiva, onde se procurou os temas referidos anteriormente. Novamente a presença dos temas descritos ao longo das ementas, objetivos, conteúdos programáticos e referências dos programas foi contabilizada, originando a tabela 2 .
Tabela 2: Relação de temas encontrados nos programas de disciplinas por Curso de Medicina. Rio de Janeiro, 2011

\begin{tabular}{lcccc}
\hline Temas Encontrados & MK & MX & MY & Total \\
\hline Políticas Públicas & 0 & 5 & 2 & 7 \\
SUS & 0 & 5 & 1 & 6 \\
Integralidade da & 0 & 5 & 1 & 6 \\
Assistência & & & & \\
Direitos Humanos & 0 & 0 & 0 & 0 \\
Sexuais e Reprodutivos & & & & \\
Estatuto da Criança e do & 0 & 0 & 0 & 0 \\
Adolescente & & & 7 & 7 \\
Gênero/Sexualidade & 0 & 0 & & \\
Ações/ Práticas & 0 & 0 & 2 & 2 \\
Educativas & 2 & 3 & 1 & 6 \\
DST/AIDS & 0 & 0 & 1 & 1 \\
Planejamento Familiar & 0 & 2 & 0 & 2 \\
Humanização da & 0 & 0 & 0 & 0 \\
Assistência & 0 & 0 & 0 & 0 \\
Direito à Saúde & 0 & 0 & 0 & 0 \\
Direito das Mulheres & 1 & 0 & 0 & 1 \\
Direito dos Adolescentes & 0 & 2 & 0 & 2 \\
Aborto & 0 & 1 & 0 & 1 \\
PNAISM & 0 & 1 & 0 & 1 \\
PAISM & 0 & 1 & 0 & 1 \\
PROSAD & 0 & 0 & 0 & 0 \\
Pacto pela Saúde & 0 & 0 & 0 & 0 \\
Lei Orgânica da Saúde & 3 & 25 & 15 & 43 \\
Constituição Federal & & \\
Total & & &
\end{tabular}

Os 20 temas listados foram encontrados 43 vezes nos 18 programas analisados. $\mathrm{O}$ tema DHSR e a ECA não são citados diretamente em nenhum programa de disciplina.

Apenas dois programas da área de Saúde da Mulher da curso MK foram selecionados para análise e os únicos temas encontrados foram DST e aborto. Porém, esses estavam inseridos no contexto hospitalar, não sendo tratados dentro da perspectiva dos DHSR. Não foi encontrada nenhuma publicação do Ministério da Saúde nesses programas.

No curso MX, 12 programas participaram da análise, sendo quatro de Saúde do Adolescente, seis de Saúde Coletiva e dois programas referentes à área de Saúde da Mulher. O curso não apresenta programa de disciplina específico para a última área, no entanto o conteúdo é ministrado em outra disciplina e citado em seus programas. Os programas de Saúde do Adolescente apresentaram aspectos voltados para patologias e síndromes no período 
da infância e adolescência. O Programa de Atenção Integral à Saúde do Adolescente (PROSAD) e o Programa de Atenção Integral à Saúde da Mulher (PAISM) são citados nas referências de um programa de Saúde Coletiva. O Pacto pela Saúde e o PNAISM, também são citados em outro programa dessa mesma área.

Dos quatro programas pertencentes ao curso MY, dois são de Saúde da Mulher e dois de Saúde Coletiva. Um dos programas de Saúde Coletiva trata diretamente do tema Gênero/Sexualidade, abrangendo aspectos biológicos, éticos e psicossociais do tema, o que explica a aparição do termo por 7 vezes. Há aspectos relacionados ao DHSR, já que propõe o desenvolvimento da prática médica sem juízo de valores, respeitando o direito do paciente e menciona termos como identidade e papéis sexuais, homossexualismo, sexualidade e planejamento familiar, gravidez, sexualidade e doenças, terapia sexual. O mesmo traz a temática Adolescente em seu conteúdo, com tópicos sobre sexualidade infantil, na puberdade e adolescência.

Apenas uma publicação do Ministério da Saúde sobre Saúde da Mulheré citada nas referências de um programa do curso MY, contudo, não se trata da Política nem do Programa de Atenção à Mulher, e está presente em um programa da área de Saúde Coletiva. As Referências dos programas da área de Saúde da Mulher só indicam livros sobre ginecologia e obstetrícia.

Novamente observa-se divergência entre a presença dos temas nos programas de disciplinas quando comparado com as referências dos mesmos. Nota-se a escassez de documentos indicados para leitura nos programas de Medicina, pois a presença dos temas cai de 43 para 16. No geral, as referências apresentam-se desatualizadas e com conteúdo de aspecto biológico, majoritariamente, reiterando a situação de Enfermagem. Entretanto, nos programas de Enfermagem a lista de referências muitas vezes era extensa, ao contrário de Medicina, onde alguns programas sequer as continham.

\section{DISCUSSÃO}

A garantia dos direitos sexuais e reprodutivos no Brasil foi posta em discussão por grupos feministas e de mulheres que, principalmente nas décadas de 1960 e 1970, organizaram-se em torno das problemáticas do gênero, da sexualidade e da reprodução, considerados temas vitais para a construção da autonomia das mulheres em relação às decisões que envolvem seus próprios corpos. A Constituição Federal de 1988 permitiu que transformações nesse âmbito ocorressem e, em torno dela, foram e são geradas políticas públicas, instrumentos legais e decisões judiciais para responder a tais demandas ${ }^{(8)}$. Os tratados internacionais de direitos humanos também foram decisivos na inserção dos DSR sob a perspectiva de direitos humanos e, principalmente, na definição de maneira mais autônoma dos direitos sexuais em relação aos direitos reprodutivos.

O Ministério da Saúde elaborou, em 2005, uma série intitulada Direitos Humanos e Direitos Reprodutivos com o objetivo de apoiar as ações de saúde sobre estes temas realizados pelos serviços de saúde: Caderno n. 1 - Direitos Sexuais e Direitos Reprodutivos: uma prioridade do governo; Caderno n. 2 - Cartilha sobre Direitos Sexuais, Direitos Reprodutivos e Métodos Anticoncepcionais; Caderno n. 3 - Anticoncepção de Emergência: perguntas e respostas para profissionais de saúde; Caderno n. 4 Norma Técnica Atenção Humanizada ao Abortamento; Caderno n. 5 - Manual Técnico Pré-Natal e Puerpério: atenção qualificada e humanizada; Caderno n. 6 - Norma Técnica Prevenção e Tratamento dos Agravos Resultantes da Violência Sexual contra Mulheres e Adolescentes; Caderno n. 7 - Aspectos Jurídicos do Atendimento às Vítimas de Violência Sexual: perguntas e respostas para profissionais de saúde ${ }^{(9)}$.

No entanto, das publicações citadas acima, apenas uma delas foi identificadaneste estudo. Destaca-se também a ausência do Caderno de Atenção Básica - Saúde Sexual e Saúde Reprodutiva ${ }^{(10)}$. Por meio do material analisado podemos inferir que temas muito presentes no cotidiano dos serviços de saúde, com relação direta na atenção ao pré-natal e à contracepção, por exemplo, estão distantes da formação profissional, indicando um distanciamento do SUS como ordenador da formação em saúde, como previsto no artigo 200, inciso III da Constituição Federal de $1988^{(11)}$.

Um estudo que analisou a abordagem sobre sexualidade, gênero e direitos e sua aplicação pedagógica por enfermeiras que desenvolviam grupos educativos em contracepção, constatou que os grupos apresentaram características bastante diversificadas em razão da forma como os temas eram abordados por cada coordenador. Constatou-se que valores culturais, geracionais e individuais interferem na incorporação e problematização de questões do campo da saúde sexual e reprodutiva. Este trabalho apontou a necessidade de mudança nos discursos e nas práticas profissionais, para isso, a discussão sobre DHSR deve ser considerada e incorporada pelos envolvidos na formação profissional em saúde ${ }^{(12)}$.

No que se refere à atenção ao Adolescente também observamos esta dissonância entre o que orienta o Ministério da Saúde e o encontrado nos programas de disciplinas. O PROSAD, documento marco na atenção a este grupo, 
está presente em apenas dois programas de dois cursos de Enfermagem e um programa na Medicina. O ECA, principal marco nacional de direitos dos adolescentes, assegura direitos universais e integrais a crianças e adolescentes $^{(13)}$. O Marco Teórico e Referencial - Saúde Sexual e Saúde Reprodutiva de Adolescentes e Jovens, publicado em 2006, destaca que os DHSR não estão previstos no Estatuto, entretanto, alguns de seus mecanismos criam pressupostos fundamentais para que eles sejam assegurados na assistência à saúde, através do reconhecimento dos adolescentes como sujeito de direitos ${ }^{(14)}$.

Além disso, em 2010, o Ministério da Saúde propôs as Diretrizes Nacionais para a Atenção Integral à Saúde de Adolescentes e de Jovens na Promoção, Proteção e Recuperação da Saúde. Essas são baseadas na Política Nacional de Atenção Integral à Saúde de Adolescentes e Jovens, aprovada em 2007, abordando a importância da formação de estratégias que contribuam para o suprimento das necessidades dessa população e para a modificação do quadro de vulnerabilidade desse grupo $^{(15)}$.

Entretanto, esses e outros documentos são pouco identificados como referências bibliográficas nos programas de disciplinas dos cursos. Apontando, assim, para um conteúdo predominante de caráter biomédico oferecido pelas disciplinas, onde ainda é muito presente a concepção restrita de saúde, associada apenas à ausência de doenças orgânicas. Este panorama também foi identificado em pesquisa semelhante em escolas de Enfermagem privadas do Município do Rio de Janeiro ${ }^{(16)}$.

Diante das conquistas legais e políticas, é imprescindível que os profissionais de saúde tenham conhecimento das mesmas e atuem de forma a torná-las uma realidade na prática de atenção à saúde. Para tanto, se faz necessária a reorientação do processo de formação de enfermeiros e médicos, assim como de outros profissionais de saúde, de modo a formar profissionais que prestem assistência à saúde da população de forma integral, respeitando os princípios e diretrizes do SUS e as orientações técnicas preconizadas por tais documentos.

Com base nas Diretrizes Curriculares Nacionais do Curso de Graduação em Enfermagem, e também de Medicina, durante a formação deve-se garantir que os futuros profissionais adquiram competências específicas de forma que reconheçam a saúde como direito, assegurando a integralidade e humanização no atendimento ${ }^{(6)}$. Porém, na prática profissional, essas competências nem sempre são exercidas devido ao distanciamento dos conteúdos curriculares necessários à formação de um profissional de saúde com perfil capaz de responder às necessidades da população ${ }^{(17)}$.
Em geral, os profissionais de saúde encontram dificuldades em abordar os aspectos relacionados à saúde sexual, na medida em que essa questão está marcada por preconceitos e tabus enraizados pela sociedade. As ações nesse campo têm sido focadas na atenção à saúde reprodutiva, voltadas para a mulher adulta e não proporcionam o envolvimento dos homens nesse meio. A reorganização da estrutura curricular nos cursos de graduação se mostra necessária e urgente para formar enfermeiros e médicos. Esses conscientes e capazes de respaldar as suas ações e exercer os direitos humanos sexuais e reprodutivos durante sua atuação nos serviços, promovendo uma mudança do atual panorama em que se encontra atenção à saúde brasileira.

\section{CONSIDERAÇÕES FINAIS}

Conclui-se que os termos DHSR e ECA não se fazem presentes nos programas, a não ser pela referência de uma publicação do Ministério da Saúde sobre DSR em apenas um programa e indicação do ECA nas referências de três programas de Enfermagem. Os temas não foram encontrados em nenhum programa de Medicina.

A partir da análise dos documentos selecionados, apurou-se a existência de temas afins aos DHSR, como planejamento familiar, aborto, DST/AIDS, direito das mulheres, direito dos adolescentes, humanização, entre tantos outros, que poderiam ser mais explorados pelos cursos, na medida em que podem ser utilizados como meio de discussão sobre os direitos humanos sexuais e reprodutivos. Contudo, a presença destes também é mínima nos programas de disciplinas. Nota-se a falta de padronização dos programas, assim como se faz necessário a revisão e atualização das suas referências. Muitos programas não apresentam os temas buscados em sua íntegra, indicando apenas assuntos relacionados a eles.

Outro aspecto observado é que nem sempre os itens analisados em um programa - ementa, objetivos, conteúdo programático e referências - tinham relação entre si. De alguma forma, isso poderá refletir na futura prática profissional desses alunos, visto que tais temáticas são de suma importância na formação profissional em saúde para que suas ações contribuam para a promoção à saúde e melhoria da qualidade de vida da população.

\section{REFERÊNCIAS}

1. Petchesky RP. Rights and needs: rethinking the connections in debates over reproductive and sexual rights. Health and Human Rights; 2000;4(2):17-29. 
2. Rosas CF. Política Nacional de Direitos Sexuais e Direitos Reprodutivos: rompendo velhos preconceitos e construindo novos paradigmas. Jornal da Rede Feminista de Saúde. 2005 set; 27:18-20.

3. Pereira AL. Ações educativas em contracepção: teoria e prática dos profissionais de saúde [tese]. Rio de Janeiro (RJ): Universidade do Estado do Rio de Janeiro; 2008.

4. Ministério da Saúde(BR). Política Nacional de Atenção Integral à Saúde de Adolescentes e de Jovens [versão preliminar]. Brasília: Ministério da Saúde; 2007.

5. Nogueira MI. As mudanças na educação médica brasileira em perspectiva. Reflexões sobre a emergência de um novo estilo de pensamento. Rev. bras. educ. med. 2009;33(2):262-70.

6. Ministério da Educação (BR). Secretaria de Educação Superior. Diretrizes curriculares para os cursos de graduação. Resolução CNE/CES n. 3, de 7 de novembro de 2001. Brasília: Ministério da Saúde; 2001.

7. Bardin L. Análise de conteúdo. Lisboa: Edições 70; 2006.

8. Vianna ARB, Carrara S. Os direitos sexuais e reprodutivos no Brasil a partir da "Constituição Cidadã". In: Oliven R, Ridenti M, Brandâo G, organizadores. A Constituição de 1988 na vida brasileira. São Paulo: Aderaldo \& Rothschild, Anpocs; 2008. p. 334-59.

9. Ministério da Saúde (BR). Secretaria de Atenção à Saúde. Direitos Sexuais e Direitos Reprodutivos: uma prioridade do governo. Brasília: Ministério da Saúde; 2005.

10. Ministério da Saúde (BR). Secretaria de Atenção à Saúde. Departamento de Atenção Básica. Saúde sexual e saúde reprodutiva. Brasília: Ministério da Saúde; 2010.

11. Brasil. Constituição da República Federativa do Brasil. Brasília: Senado; 1988.

12. Lemos A. Grupos educativos em contracepção: narrativas e práticas de enfermeiras. Cogitare enferm. 2011;16(1):36-42.

13. Ministério da Ação Social, Justiça, Trabalho e Educação (BR). Lei n. 8.069, de 13 de julho de 1990. Dispõe sobre o Estatuto da Criança e do Adolescente e dá outras providências. Brasília; 1990.

14. Ministério da Saúde (BR). Marco teórico e referencial: saúde sexual e saúde reprodutiva de adolescentes e jovens. Brasília: Ministério da Saúde; 2006.

15. Ministério da Saúde (BR). Diretrizes Nacionais Para a Atenção Integral à Saúde de Adolescentes e Jovens na Promoção, Proteção e Recuperação da Saúde. Brasília: Ministério da Saúde; 2010.

16. Carvalho JO, Lemos A, Cerqueira JC. Direitos humanos sexuais e reprodutivos. Um tema na formação do enfermeiro [TCC]. Rio de Janeiro; 2011.

17. Feuerwerker LCM, Ceccim RB. O quadrilátero da formação para a área da saúde. Ensino, gestão, atenção e controle social. Physis. 2004;14(1):41- 65.

Cogitare Enferm. 2013 Jan/Mar; 18(1):64-70 